\title{
RUPTURED INTERSTITIAL PREGNANCY ON RIGHT FALLOPIAN TUBE
}

\author{
Hemalatha Devi ${ }^{1}$, Rajya Lakshmi²
}

\section{HOW TO CITE THIS ARTICLE:}

Hemalatha Devi, Rajya Lakshmi. "Ruptured interstitial Pregnancy on right Fallopian Tube". Journal of Evolution of Medical and Dental Sciences 2015; Vol. 4, Issue 06, January 19; Page: 1063-1067,

DOI: $10.14260 /$ jemds/2015/149

ABSTRACT: Ectopic pregnancy is the leading cause of mortality in early pregnancy, the incidence of ectopic pregnancy is estimated to be between 1-2\%. The majority of these pregnancies are located in the fallopian tube. Rarely interstitial pregnancy implanted in the interstitial part of the fallopian tube. It is associated with higher mortality due to massive intra peritoneal haemorrhage. This data collected from the government general hospital, Rangaraya medical college. The incidence of interstitial pregnancies is $2-4 \%$ of all tubal pregnancies or 1 in 3000 to 5000 live births. This case presented to our labour room. She was a 33 years old lady G4P2L2A1 with 4 months amenorrhoea with severe pain abdomen and giddiness. General examination showed she is a conscious and coherent. Grossly pale with haemoglobin of 4 grams, blood pressure of $80 / 60 \mathrm{mmHg}$. There was abdominal distension, tenderness and guarding. pervaginal examination showed tender cervical movements with fullness of pouch of douglas, exact size of uterus could not be made out. Case was diagnosed as ruptured ectopic gestation on right side. Ultra sound suggestive of ruptured ectopic pregnancy. Patient was taken for emergency laparotomy haemoperitonium of $2500 \mathrm{ml}$ noted fetus of 14 weeks with intact gestation sac seen in the peritoneal cavity, ruptured noted on the right cornual region, cornual resection and rent repair was done along with right Salpingo oophorectomy. Postoperative period uneventful.

KEYWORDS: Interstitial, ectopic, haemoperitomium, cornual resection.

INTRODUCTION: Ectopic pregnancy is the leading cause of mortality in early pregnancy, the incidence of ectopic pregnancy is estimated to be between 1-2\%. The majority of these pregnancies are located in the fallopian tube. Risk factor includes previous pelvic inflammatory disease, IUCD usage endometriosis and assisted reproductive technology.

This Interstitial pregnancy implanted intra murally in the interstitial part of the fallopian tube.

It is the rarest variety of tubal pregnancy because of the thick and vascular musculature of uterine wall with greater distensibility the fetus grows dissecting the muscle fibre for a longer period (12-14 weeks) before rupture.

It is associated with higher mortality than other tubal pregnancies due to massive intra peritoneal haemorrhage. Diagnosis before rupture is very difficult

Cornual pregnancy occurs in a rudimentary horn or in one horn of bicornuate uterus.

The terms interstitial and cornual pregnancies have been used interchangeably.

CASE REPORT: A 33 year old lady G4P2L2A1 presented to the emergency labour room with 4 months amenorrhoea, severe pain abdomen and giddiness. She had intermittent pain abdomen for the last 4 months relieved on medication. 
Obstetric history revealed all full term normal deliveries. In the third pregnancy, that is 4 years back she underwent laparotomy with left salpingectomy for ruptured ectopic for 6 weeks period of amenorrhea and right side tubectomy was done. Previous menstrual cycles are regular. General examinations showed she is conscious and coherent. Grossly pale with haemoglobin of 4 grams. Pulse rate 130 per minute, blood pressure $80 / 60 \mathrm{mmHg}$.

Per abdominal examination showed abdominal distension, tenderness and guarding. bowel sounds absent.

Pervaginal examination showed tender Cervical movements with fullness of pouch of douglas, exact size of uterus could not be made out.

INVESTIGATIONS: urine pregnancy test positive, haemoglobin 4 grams \%, B+ve blood group, renal parameters within normal limits, blood sugar $70 \mathrm{mg} / \mathrm{dl}$, ultra sound abdomen revealed collection of fluid in the abdominal cavity and Douglas pouch, bulky uterus, gestational sac with fetus of 14 weeks size seen in the right adenexal region. Ultra sound suggestive of ruptured ectopic pregnancy.

\section{MANAGEMENT:}

- The patient consent was taken for emergency laparotomy.

- Emergency laparotomy done under general anaesthesia.

- Haemoperitoneum of $2500 \mathrm{ml}$ noted, fetus of 14 weeks with intact gestational sac seen in the peritoneal cavity, rupture noted on the right cornual region, cornual resection and rent repair was done along with right salpingo oophorectomy. Left fallopian tube is absent.

- Haemostatis secured. Intra operatively, 2 units of blood transfusion were given. Another 2 units of blood transfusion given during post-operative period. Her post-operative period was uneventful. She was followed for two consecutive months and she did not have any complaints.

DISCUSSION: An interstitial pregnancy is a rare form of ectopic pregnancy which is implanted in the interstitial part of the fallopian tube, while interstitial pregnancies account for only 2-3\% of all ectopic pregnancies. It progressed to 12-14 weeks asymmetrically In contrast to other tubal pregnancies. Ipsilateral salpingectomy, previous ectopic pregnancy, and in vitro fertilization are predisposing factors for interstitial pregnancy.[1] In this case she underwent salpingectomy with opposite side tubectomy for previous ectopic gestation.

Interstitial segment of the tube lies close to the uterine vessels hence they cause a disproportionately high incidence of haemoperitonium and shock. And the mortality rate is approximately twice that of other types of ectopic pregnancies. ${ }^{[2]}$

The terms interstitial and cornual pregnancies have been used interchangeably but the term cornual pregnancy usually denotes gestation in one horn of bicornuate or septate uterus. The interstitial portion of the fallopian tube is highly vascular with thick muscular wall this allows for the gestation to advance much further before rupture takes place with excessive intra peritoneal haemorrhage. Incidence of ectopic following tubal ligation within 10 years. Is 7/1000.

Difficult to diagnose inerstitial pregnancy leading to catastrophic situation.

Typical symptoms include abdominal pain, vaginal bleeding, haemorrhagic shock is found in almost a quarter of patients. 


\section{CASE REPORT}

The sonographic criteria for the diagnosis of interstitial pregnancy include:

1. An empty uterine cavity.

2. Gestation sac seen separately and more than $1 \mathrm{~cm}$ from the most lateral edge of the uterine cavity.

3. A thin myometrial layer $(<5 \mathrm{~mm})$ surrounding the chorionic sac.

4. Typically, the interstitial line sign - an echogenic line from the endometrial cavity to the corner next to the gestational mass is seen.[3]

- A suggestive but nonspecific sign is an eccentrically located gestational sac.

- The interstitial pregnancy was confirmed during laparoscopy.

- The conventional treatment of interstitial pregnancy has been cornual resection or hysterectomy.

\section{MANAGEMENT:}

\section{SURGICAL LAPAROTOMY:}

- Hysterectomy.

- Cornual resection.

- Uterine artery ligation and repair of ruptured uterine corneal.

\section{LAPAROSCOPIC PROCEDURES:}

- Cornual resection.

- Salpingostomy

- Cornual resection and salpingectomy

- Endoloop and encircling suture25

\section{MANAGEMENT IN UNRUPTURED ECTOPIC:}

\section{MEDICAL MANAGEMENT:}

- Systemic methotrexate.

- USS guided methotrexate.

- Laparoscopic guided methotrexate/potassium chloride.

- Systemic methotrexate followed by selective uterine artery.

- Embolization

\section{OTHER CONSERVATIVE SURGERY BY HYSTEROSCOPIC PROCEDURES:}

- Hysteroscopic endometrial resection under laparoscopic control.

- Hysteroscopic cornual evacuation aided by polyp forceps under ultrasound (USS) or laparoscopic guidance.

Early diagnosis of interstitial pregnancy has made various conservative and less invasive management techniques feasible.

Laparoscopic approach for cornual resection or salpingotomy via myometrium has been reported. ${ }^{[4]}$ Blood loss can be minimized by doing Electro coagulation of ascending branch of uterine artery. Conservative management of interstitial pregnancy using injection potassium chloride or methotrexate into the gestational sac has been successfully achieved.[5] 
Weekly follow up with beta HCG levels and ultra sound examination are important as low and declining beta HCG levels are not always associated with resolution of ectopic gestation and rupture can occur during conservative management.

Routine ultra sound done at 10-13 weeks in all antenatal clinics may help in early diagnosis that is before rupturing. This will decrease morbidity and mortality associated with rapid and massive haemoperitonium due to rupture:

- Treatment is exision of cornual end since the sac is present on the upper part of uterus.

- It is important to avoid pregnancy for atleast 1 year.

- Investigations necessary are ultra sound, serum beta HCG and blood group and typing.

- MRI can be used to differentiate interstitial pregnancy from angular pregnancy.

CONCLUSION: Suspect Ruptured Ectopic pregnancy when women comes with history of amenorrhoea with pain abdomen with shock. Diagnosis confirmed by ultra-sound and urine pregnancy test or serum beta HCG levels. Laparotomy or laparoscopic cornual resection or hysterectomy was the treatment of choice in a ruptured interstitial pregnancy.

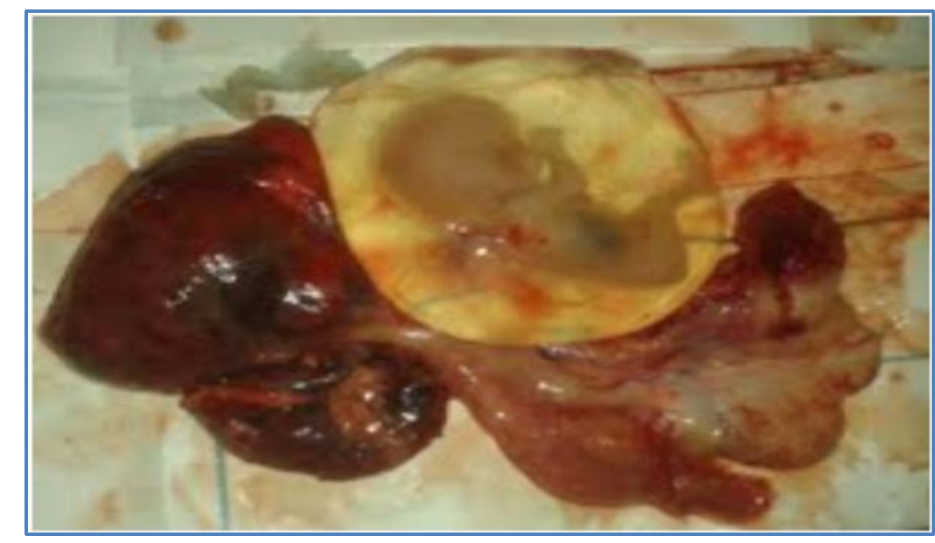

Cornual resection with Right salpingo opharectomy

\section{REFERENCES:}

1. Tulandi, T.; Al-Jaroudi, D. (2004). "Interstitial pregnancy: results generated from the Society of Reproductive Surgeons Registry". Obstetrics \& Gynecology 103 (1): 47-50. doi: 10.1097/01.AOG.0000109218.24211.79. PMID 14704243.

2. Maliha WE, Gonella P, Degnan EJ. Ruptured interstitial pregnancy presenting as an intrauterine pregnancy by ultrasound. Ann Emerg Med. 1991; 20: 910-2. [PubMed]

3. Ackerman, T. E.; Levi, C. S.; Dashefsky, S. M.; Holt, S. C.; Lindsay, D. J. (1993). "Interstitial line: sonographic finding in interstitial (cornual) ectopic pregnancy". Radiology 189 (1): 83-87. PMID 8372223.

4. Soriano, D.; Vicus, D.; Mashiach, R.; Schiff, E.; Seidman, D.; Goldenberg, M. (2008). "Laparoscopic treatment of cornual pregnancy: a series of 20 consecutive cases". Journal of Reproductive Immunology 90 (3): 839-843. doi:10.1016/j.fertnstert.2007.07.1288. PMID 17936282.

5. Lau, S.; Tulandi, T. (1999). "Conservative medical and surgical management of interstitial ectopic pregnancy". Fertil Steril 72 (2): 207-215. 


\section{AUTHORS:}

1. Hemalatha Devi

2. Rajya Lakshmi

\section{PARTICULARS OF CONTRIBUTORS:}

1. Associate Professor, Department of Obstetrics and Gynaecology, Rangaraya Medical College. Kakinada, Andhra Pradesh.

2. Professor, Department of Obstetrics and Gynaecology, Rangaraya Medical College, Kakinada, Andhra Pradesh.

\author{
NAME ADDRESS EMAIL ID OF THE \\ CORRESPONDING AUTHOR: \\ Dr. Hemalatha Devi, \\ \# D-14, Doctors Quarters, \\ Maharanipeta, Visakhapatnam, \\ Andhra Pradesh-530002. \\ E-mail: hema.vgh.gyn@gmail.com
}

Date of Submission: 29/12/2014.

Date of Peer Review: 30/12/2014.

Date of Acceptance: 09/01/2015.

Date of Publishing: 19/01/2015. 